\title{
ACIDEZ Y PESO ESPECÍFICO DE LA LECHE DE CABRA DE UN GRUPO DE CAPRICULTORES DE LA MESETA CENTRAL COSTARRICENSE ${ }^{1}$
}

\author{
Alejandro Chacón Villalobos ${ }^{2}$
}

\begin{abstract}
RESUMEN
Acidez y peso específico de la leche de cabra de un grupo de capricultores de la Meseta Central costarricense. El estudio fue efectuado en la Estación Experimental Alfredo Volio Mata de la Universidad de Costa Rica y en la Asociación Costarricense de Criadores de Cabras localizadas en el Alto de Ochomogo de Cartago. La leche de cabra de siete productores fue evaluada desde el primero de junio hasta el 31 de diciembre de 2002. El peso en $\mathrm{kg}$, la acidez en grados Dornic y el peso específico de la leche fueron registrados diariamente. Se determinó un valor característico de $17,7 \pm 0,3^{\circ} \mathrm{D}$ para la acidez y de 1,029 $\pm 0,002$ para el peso específico. Análisis de varianza seguidos de una prueba post hoc de Scheffé se efectuaron para evaluar posibles variaciones en la acidez o el peso específico a lo largo de los meses para todos los datos sin importar el origen de la leche. Igualmente se evaluaron posibles variaciones en las mismas variables para cada uno de los productores y entre ellos a lo largo de los meses. Los resultados obtenidos en todos los casos mostraron que no existieron diferencias en todas las determinaciones evaluadas para acidez $(\mathrm{P} \leq 0,05)$. Para el peso específico si se detectaron diferencias significativas $(\mathrm{P} \leq 0,01)$ en todas las determinaciones evaluadas. Al correlacionar el peso específico y la acidez de todos los datos se obtuvo un coeficiente de correlación de 0,03 , lo cual hace imposible argumentar la existencia de alguna relación proporcional entre estas dos variables. Al correlacionar la cantidad de leche producida con el peso específico se obtuvo un coeficiente de correlación de 0,67 .
\end{abstract}

Palabras claves: leche de cabra, acidez, peso específico,

\begin{abstract}
Acidity and specific gravity of goat milk produced by a group of breeders from the Costa Rican Central Plateau. The evaluation was conducted at the Alfredo Volio Experimental Station of the University of Costa Rica and at the Dairy Processing Facility of the Costa Rican Association of Goat Breeders (A.C.C.C) both located in Ochomogo, Cartago. Goat milk supplied by the seven producers was evaluated from June $1^{\text {st }}$ to December $31^{\text {th }}$ of 2002. Weight, acidity expressed as Dornic units, and specific gravity of the milk brought to the facility by every producer were measured daily. From the total data that was gathered, a characteristic value of $17,7 \pm 0,3{ }^{\circ} \mathrm{D}$ for acidity and 1,029 $\pm 0,002$ for specific gravity were determinated, both for a confidence level of $99 \%$. ANOVA statistical essays were conducted followed by Scheffé post hoc test in order to study the variation of acidity and specific gravity at different months for all the data regardless of the producer. In the other hand variations on both parameters for each producer and among them over the moths were also studied by the statistical methodology mentioned above. The results obtained showed no significant differences in acidity for any of the evaluations $(\mathrm{P} \leq 0,05)$, however significant variations were detected for specific gravity for the same set of tests. When correlated, specific gravity and acidity showed a very low correlation coefficient of 0,03 which makes impossible to argue any sort of relation between both variables. The same happened when the weight of milk brought by every producer day by day was correlated with specific gravity $(r=-0,67)$
\end{abstract}

Key words: goat milk, acidity, specific gravity, quality.

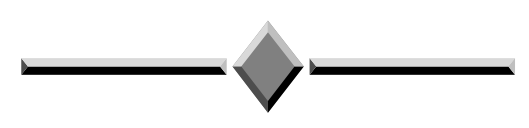

\section{INTRODUCCIÓN}

La leche es un producto que se altera con mucha facilidad, ya sea por acción microbiana, por manipula- ción deshonesta o por tratamiento industrial. Su sabor tiende a ser dulcete debido a la lactosa que contiene, la cual es el principal sustrato de acción microbiana fermentativa (Nasanovsky et al. 2003).

\footnotetext{
1 Recibido para publicación el 10 de marzo del 2004. Inscrito en la Vicerretoría de Investigación de la Universidad de Costa Rica, Proyecto $\mathrm{N}^{\mathrm{o}}$ 737-98-005.

2 Estación Experimental Alfredo Volio Mata. Facultad de Ciencias Agroalimentarias. Universidad de Costa Rica. achacon@cariari.ucr.ac.cr.
} 
La acidez de la leche es un criterio muy importante de calidad. Por lo general, posee un valor de $\mathrm{pH}$ (reacción iónica) cercano a la neutralidad y que varía entre 6,5 y 6,65 (Alais 1988). La acidez porcentual expresada como ácido láctico ronda valores de $0,15 \%$ a $0,16 \%$ en la leche fresca, tolerándose un máximo de un $0,18 \%$ (Herrera, 1995). En la industria, la acidez de la leche puede medirse en grados Dornic $\left({ }^{\circ} \mathrm{D}\right)$, donde un valor del $19^{\circ} \mathrm{D}$ es comúnmente el máximo de acidez tolerable para el procesamiento posterior de la leche (Chacón 2003).

Los valores de acidez pueden variar drásticamente en leches contaminadas, dado que los microorganismos forman ácido láctico a partir de la lactosa, disminuyendo así el pH y aumentando la acidez (Herrera 1995). Por otro lado, la leche de muy baja acidez puede ser sinónimo de mastitis en el animal que la produjo. El valor de acidez es por lo tanto un indicador de la contaminación microbiana.

El peso específico es otra medida de calidad importante en la leche y corresponde a una medida relativa entre la densidad de la leche y la densidad del agua a la misma temperatura de medición, generalmente de $15,5^{\circ} \mathrm{C}\left(60^{\circ} \mathrm{F}\right)$ (Herrera 1995). La leche íntegra ronda valores extremos de peso específico entre 1,028 y 1,034 mientras que la leche descremada suele tener mayor peso específico que 1,034 (Nasanovsky et al. 2002). Al estar el peso específico ligado con la densidad, esta es una medida indirecta de la concentración de sólidos en la leche. Una leche con un peso específico menor a 1,028 puede ser un indicador de adulteración con agua (Chacón 2003).

Idealmente para el procesamiento de la leche se requiere que esta sea íntegra y no mezclada con agua. Además, es importante que la misma no se encuentre deteriorada y contaminada. En la pequeña industria, donde muchas veces la leche no se produce bajo la supervisión de una planta industrial, no se puede estar seguro de la calidad, a no ser que se efectúen unas simples pruebas de evaluación preliminares. Estas pruebas son en la mayoría de los casos la determinación de la acidez de la leche y la determinación del peso específico (Chacón 2003).

Los objetivos de este ensayo fueron caracterizar la calidad de la leche de cabra producida por siete capricultores de la Meseta Central costarricense de modo tal, que se pudiese evaluar la variación de la acidez y el peso específico durante la estación lluviosa y determinar así un valor característico. Así mismo, se estudió la existencia de alguna relación proporcional entre las variaciones de la acidez, el peso específico y los volúmenes de entrega.

\section{MATERIALES Y MÉTODOS}

El experimento se llevó a cabo entre junio y diciembre del año 2002 en las instalaciones del módulo de producción de derivados lácteos de la Asociación Costarricense de Criadores de Cabras (A.C.C.C) y en las instalaciones de la Estación Experimental Alfredo Volio Mata de la Universidad de Costa Rica, ambas ubicadas en el Alto de Ochomogo en la provincia de Cartago.

Durante un período de seis meses se registraron día a día las cantidades en kilogramos de leche entregadas por cada uno de los siete productores activos y afiliados a la A.C.C.C.

Para cada muestra de leche entregada por los productores en el período de estudio, se valoró la acidez titulable como ácido láctico expresada en la escala de Dornic $\left({ }^{\circ} \mathrm{D}\right)$. El método consistió en la titulación con indicador de fenoftaleína de una alícuota de $9,00 \mathrm{ml} \mathrm{a}$ $20{ }^{\circ} \mathrm{C}$ y empleando como valorante $\mathrm{NaOH} 0,1000 \mathrm{~N}$ según el método recomendado por Herrera (1995). El equipo volumétrico empleado fue un acidímetro marca "Gerber" graduado en la escala de acidez de Dornic.

Se determinó el peso específico para las mismas muestras simultáneamente con la prueba de acidez, todas aquellas entregadas con más de cuatro horas de ordeñadas tal y como señala Herrera (1995). La determinación se efectuó empleando un lactodensímetro de Quevenne graduado y calibrado para 60 grados Fahrenheit, y con un rango entre 20 y 40 unidades, que se puso a flotar en $400 \mathrm{ml}$ de leche depositada en una probeta de $500 \mathrm{ml}$. La lectura del lactodensímetro se corrige para aquellas muestras cuya temperatura es diferente de $60{ }^{\circ} \mathrm{F}$ restando 0,1 unidades a la lectura del aparato por cada grado menos o bien sumando 0,1 por cada grado más. La lectura final se determina por cálculo matemático a partir de la lectura corregida empleando la siguiente fórmula:

$$
\text { Peso específico }=\left(\frac{\text { Lectura corregida }}{1000}\right)+1,00
$$

El número de muestras evaluadas en acidez y pesos específico para cada proveedor dependió del número de entregas que efectuó, a razón de un análisis por entrega y sumó un total de 499 mediciones para todos los productores en conjunto. Para los datos de peso específico y de acidez obtenidos en el período experimental se efectuaron determinaciones estadísticas para el intervalo de confianza de $99 \%$ para caracterizar la media por productor siguiendo el método descrito por Haber \& Runyon (1973). La media para acidez y peso específico asumiendo todos los datos de los diferentes productores fue 
determinada estadísticamente para el intervalo de confianza de $99 \%$.

Para detectar diferencias significativas se realizaron análisis de varianza seguidos de un análisis post hoc de Scheffé para aquellas fuentes de variación que resultaron significativas $(\mathrm{P}<0,05)$ tal y como describe Chacón (2000) para grupos de variables de tamaños desiguales. Los análisis fueron para el peso específico entre meses para cada productor, semestrales entre productores y entre meses para toda la población. Se procedió igualmente para detectar diferencias significativas entre meses para cada productor y semestrales entre productores con la variable acidez.

Finalmente se evaluaron las correlaciones existentes entre la acidez de una muestra, su correspondiente peso específico y el volumen de leche entregada en ese momento para el total de los datos experimentales siguiendo el método descrito por Haber y Runyon (1973).

El análisis estadístico y gráfico se efectuó empleando el paquete estadístico SAS (SAS 1985).

\section{RESULTADOS Y DISCUSIÓN}

El Cuadro 1 muestra la cantidad total de leche entregada por cada uno de los productores a lo largo de los siete meses de estudio, así como la estimación promedio de la cantidad de leche diaria entregada y el número de entregas efectuadas.

Cuadro 1. Cantidad de leche total de cabra y estimado promedio diario de entrega para los siete productores. Ochomogo, Cartago, Costa Rica. 2002.

\begin{tabular}{cccc}
\hline Productor & $\begin{array}{c}\text { Leche total } \\
\text { entregada }(\mathbf{k g})\end{array}$ & $\begin{array}{c}\text { Promedio } \\
\text { diario }(\mathbf{k g})\end{array}$ & $\begin{array}{c}\text { Número de } \\
\text { entregas }\end{array}$ \\
\hline 1 & $1.470,9$ & 16,5 & 89 \\
2 & $1.406,1$ & 18,0 & 78 \\
3 & $1.027,6$ & 13,2 & 78 \\
4 & 455,1 & 6,2 & 73 \\
5 & 665,7 & 6,2 & 108 \\
6 & 919,7 & 21,7 & 42 \\
7 & $1.789,5$ & 57,5 & 31 \\
Total & $7.734,6$ & 15,5 & 499 \\
\hline
\end{tabular}

Los intervalos de confianza para el 99\% que fueron determinados para la acidez y el peso específico se detallan en el Cuadro 2.
Cuadro 2. Intervalos de confianza, de la acidez medida en grados Dornic $\left({ }^{\circ} \mathrm{D}\right)$ y del peso específico a lo largo del período de siete meses (por el productor). Ochomogo, Cartago, Costa Rica. 2002.

\begin{tabular}{ccc}
\hline Productor & $\begin{array}{c}\text { Acidez } \\
\text { Intervalo 99\% }\end{array}$ & $\begin{array}{c}\text { Peso específico } \\
\text { Intervalo 99\% }\end{array}$ \\
\hline 1 & $18 \pm 1$ & $1,030 \pm 0,004$ \\
2 & $16,9 \pm 0,5$ & $1,029 \pm 0,004$ \\
3 & $18,3 \pm 0,2$ & $1,030 \pm 0,005$ \\
4 & $17,2 \pm 0,4$ & $1,029 \pm 0,004$ \\
5 & $17,8 \pm 0,3$ & $1,029 \pm 0,004$ \\
6 & $14,4 \pm 0,5$ & $1,030 \pm 0,006$ \\
7 & $16,9 \pm 0,6$ & $1,029 \pm 0,007$ \\
\hline
\end{tabular}

Para la acidez, puede concluirse que la media, asumiendo todos los datos de los productores, fue para el intervalo de confianza de $99 \%$ de $17,7 \pm 0,3^{\circ} \mathrm{D}$ con una desviación estándar de 2,04. En el caso del peso específico es posible concluir que el valor característico fue de $1,0299 \pm 0,0002$ para un $99 \%$ de confianza con una desviación estándar de 0,001 .

Los resultados enunciados son congruentes y característicos con aquellos citados en las fuentes bibliográficas (Herrera 1995, Nasanovsky et al. 2003), donde la leche de buena calidad debe ostentar valores de acidez por debajo de los $19^{\circ} \mathrm{D}$ y de peso específico por encima de 1,028.

La acidez, al ser un parámetro relacionado con el deterioro microbiano, es de esperar que guarde una relación directa con la manipulación y buen manejo de la materia prima antes de su llegada a la planta. Ello justificaría que sea más propenso a la variación si no se tiene un adecuado manejo. Esta podría ser la explicación de la variabilidad expresada en términos de la desviación estándar de algunos productores, derivada de un mal manejo sanitario de la operación de ordeño, de almacenamiento, de distribución o todos. Lo mismo sería de sospechar en productores cuya variación fluctúe a tal grado que haga dudar de la constancia y planificación de sus buenas prácticas de recolección.

Del análisis de los datos por medio de un análisis de varianza de una sola cola con el método de corrección post hoc de Scheffé $(\mathrm{P} \leq 0,05)$, se desprende que si se comparan entre si los productores con base al total de los datos a lo largo del período de estudio, solamente el productor dos presentó leche de acidez significativamente más baja que los productores uno y tres para todo el período $(\mathrm{P} \leq 0,01)$. No obstante no es posible concluir que existan diferencias significativas entre los demás productores en el valor de acidez de la leche durante los meses evaluados $(\mathrm{P} \leq 0,05)$. 
El peso específico de la leche de cabra presentó variaciones estacionales importantes en el tiempo, siendo dichas variaciones evidentes en seis de los productores evaluados.

Si se comparan entre si los productores, con base al total de los datos a lo largo del período de estudio, para la variable peso específico, no fue posible afirmar que existieran diferencias significativas entre productores en cuanto al peso específico de la leche entregada a la planta $(\mathrm{P} \leq 0,05)$. En otras palabras no puede afirmarse que algún proveedor entregó leche significativamente diferente en peso específico que los otros a todo lo largo del período.

Al analizar la variación de la acidez entre cada mes y para cada productor individualmente con el afán de evaluar si un productor entrega leche con acidez significativamente diferente de un mes a otro, se obtiene que no es posible establecer que existan diferencias significativas en la acidez de la leche entregada en ninguno de los casos $(\mathrm{P} \leq 0,05)$.

Con base en los resultados puede establecerse que la acidez parece no ser un aspecto de alta variabilidad entre los productores y que no depende de una estacionalidad que sea perceptible, al menos en el periodo de estudio. Es de esperar que muy posiblemente las variaciones de la acidez dependan más de las buenas prácticas de ordeño que de la naturaleza misma del animal que la produce y de su ciclo (Alais 1988). Indirectamente afianza la credibilidad del método de medición de la acidez titulable como prueba de control de calidad ante la estabilidad del parámetro en el tiempo.

Si se analiza la variación del peso específico entre cada mes y para cada productor individualmente se obtiene una muy marcada variación a lo largo del período en la mayoría de los productores.

El productor uno presentó un peso específico del mes de julio (1,029 en promedio) que demuestra ser significativamente más bajo $(\mathrm{P} \leq 0,01)$ que el de los meses de setiembre $(1,030$ en promedio), octubre $(1,031$ en promedio), noviembre (1,031 en promedio) y diciembre (1,031 en promedio). Es decir, el peso específico aumentó significativamente a partir de julio de $2002(\mathrm{P} \leq 0,01)$.

El productor dos aportó leche en el mes de julio de peso específico (1,028 en promedio) significativamente más bajo $(\mathrm{P} \leq 0,01)$ que el correspondiente al mes de setiembre (1,031 en promedio). Este último mes es a su vez significativamente más alto $(\mathrm{P} \leq 0,01)$ que el mes de noviembre (1,028 en promedio).
El productor tres presentó la mayor cantidad de diferencias importantes en cuanto a peso específico a lo largo del período ( $\mathrm{P} \leq 0,01$ en todos los casos). En primer lugar, los valores medidos durante el mes de junio generan un peso específico (1,029 en promedio) que es significativamente menor al encontrado en los meses de octubre y de diciembre (1,032 en promedio). Para el mes de julio el peso específico (1,029 en promedio) es significativamente menor $(\mathrm{P} \leq 0,01)$ al de los meses de octubre, noviembre (1,031 en promedio) y diciembre. $\mathrm{El}$ mes de agosto fue a su vez significativamente menor $(\mathrm{P} \leq 0,01)$ en peso específico $(1,029$ en promedio) con respecto a octubre y diciembre. Finalmente el mes de setiembre (1,030 en promedio) fue significativamente menor $(\mathrm{P}<0,01)$ al mes de diciembre.

No fue posible establecer diferencias significativas en el peso específico de la leche entregada por el productor cuatro a lo largo del período $(\mathrm{P} \leq 0,05)$.

Para el productor cinco fue posible establecer que el peso específico del mes de junio (1,029 en promedio) fue significativamente más bajo ( $\mathrm{P} \leq 0,01)$, que el correspondiente al mes de setiembre (1,031 en promedio). Así mismo el valor correspondiente al mes de julio $(1,028$ en promedio) es significativamente más bajo $(\mathrm{P} \leq 0,01)$ que los meses de setiembre y noviembre (1,030 en promedio).

La leche aportada por el productor seis presentó igualmente variaciones significativas $(\mathrm{P} \leq 0,01)$ entre los primeros meses del período y los de finales. El peso específico del mes de junio (1,029 en promedio) fue significativamente más bajo $(\mathrm{P} \leq 0,01)$ que el del mes de diciembre (1,031 en promedio). Lo mismo ocurrió para el mes de julio (1,029 en promedio) en comparación con noviembre (1,031 en promedio) y diciembre (1,031 en promedio). Igualmente el mes de agosto presenta valores de peso específico de menor magnitud $(\mathrm{P} \leq 0,01)$ que los dos últimos meses del año (1,029 en promedio).

El productor siete entregó en julio leche de peso específico (1,029 en promedio) significativamente menor ( $\mathrm{P} \leq 0,01)$ al de diciembre (1,032 en promedio). Los meses de octubre y de noviembre $(1,029$ en promedio para ambos meses), presentan valores de peso específico que son a su vez significativamente menores en comparación al mes de diciembre $(\mathrm{P} \leq 0,01)$.

Al evaluar si existen diferencias significativas en el peso específico entre meses para el total de la leche recibida en planta sin detallar en cada productor, para los datos de todos los productores y entre meses, existieron diferencias significativas $(\mathrm{P} \leq 0,01)$ para el peso específico del mes de junio con respecto a los meses de 
setiembre, octubre, noviembre y diciembre. En otras palabras, el peso específico de la leche entregada en junio fue significativamente menor $(\mathrm{P} \leq 0,01)$ al peso específico de los meses antes mencionados. El mismo caso se dio para el mes de julio, el cuál no solo presentó un peso específico significativamente $(\mathrm{P} \leq 0,01)$ más bajo $(1,029$ en promedio) que los meses de setiembre ( 1,030 en promedio), octubre (1,030 en promedio), noviembre (1,030 en promedio) y diciembre $(1,031$ en promedio), sino que también es significativamente más bajo que el mes de agosto (1,030 en promedio). Este último mes se presentó un peso específico significativamente menor $(\mathrm{P} \leq 0,01)$ que el mes de diciembre.

Con base en los resultados puede verse que el peso específico tendió a presentar variabilidad en diferentes momentos del año y en forma generalizada entre los productores por lo cual a diferencia de la acidez, la evaluación de la calidad por medio de este factor debería tomar en cuenta esta variabilidad. Si se toma en cuenta que la leche de los diferentes productores no es significativamente diferente entre sí, puede especularse que la variación a lo largo del año es generalizada para todos ellos. No se cuenta con bibliografía que de una explicación causal al fenómeno de esta posible variación estacional. Lo que si es cierto es que de confirmarse este fenómeno en un estudio posterior más extenso, esto es de amplia importancia, pues se estaría llegando a la conclusión de que al presentar la leche de cabra diferente concentración de sólidos en diferentes épocas, los rendimientos obtenidos para los diferentes derivados lácteos no serían constantes a lo largo del año. Esto requeriría de un sistema de compra y pago de la leche basado en el contenido de sólidos y no en volumen como así se practica en la mayor parte de la industria quesera rural (Boschini 2003).

Al evaluar la posible existencia de correlación entre las variables de peso específico y acidez se obtuvo un coeficiente de correlación de 0,03 , por lo cual se puede concluir que existió una muy pobre correlación entre las variables. Es posible que aquellos sólidos fermentables o valorables de la leche representen una fracción muy reducida de los sólidos totales, como para que variaciones en su cantidad generen cambios apreciables en el peso específico.

Algunas fuentes (Boschini 2003), reportan la existencia de una cierta tendencia en el peso específico a aumentar a medida que la producción de leche de cabra disminuye. Al correlacionar la entrega mensual total de leche y el peso específico mensual promedio de cada mes se obtiene un coeficiente de $-0,67$ lo cual permite señalar la existencia de una correlación inversa de mediana magnitud. Dado que la entrega de leche de los productores generalmente representaba el $100 \%$ de su producción se asumió que una baja en la entrega correspondía a una baja en la producción.

A pesar de que la relación entre entrega mensual contra peso específico promedio fue baja, esta indica la existencia de una tendencia indefinida. Estudios más detallados podrían efectuarse para la corroboración más exhaustiva de este supuesto.

\section{LITERATURA CITADA}

ALAIS, C. 1988. Ciencia de la leche. México. Ed. Continental.400 p.

BOSCHINI, C. 2003 Comportamientos estacionales de la composición de la leche. Ochomogo, Cartago. Comunicación Personal.

CHACÓN, A. 2000. Estudio de la maduración, la inyección de cloruro de calcio, la cocción y el congelamiento como fundamentos de un proceso para el mejoramiento de la suavidad del solomo. Tesis Licenciatura en Tecnología de Alimentos. Escuela de Tecnología de Alimentos, Facultad de Agronomía. Universidad de Costa Rica. 176 p.

CHACÓN, A. 2003. La elaboración del queso fresco y otros derivados lácteos: guía básica artesanal y de la pequeña industria. San Pedro de Montes de Oca, San José. Ed. Universidad de Costa Rica. 66 p.

HABER, A.; RUNYON, R. 1973. Estadística General. Massachusetts, Estados Unidos de América. Ed. Fondo Educativo Interamericano. $371 \mathrm{p}$.

HERRERA, C. 1995. Manual de Laboratorio de Química de Alimentos San Pedro de Montes de Oca, San José. Ed. Escuela de Quimica Universidad de Costa Rica. 100 p.

NASANOVSKY, M; GARIJO, R.; KIMMICH, R. 2003. Lechería. Estados Unidos de América. Ed. Fondo Educativo Interamericano. $49 \mathrm{p}$.

SAS. 1985. Statistical analysis system. SAS User`s Guide; Statistics 5 ed. SAS Institute Inc. Cary, NC, 373 p. 